\title{
Idiopathic pulmonary fibrosis: multiple causes and multiple mechanisms?
}

\author{
T.M. Maher*\#, A.U. Wells* and G.J. Laurent*
}

ABSTRACT: Idiopathic pulmonary fibrosis (IPF) is a devastating condition that carries a prognosis worse than that of many cancers.

A recent classification of the idiopathic interstitial pneumonias has redefined the diagnostic criteria necessary to determine a diagnosis of IPF. The present authors believe that this redefinition is incorrect, relying as it does on subtle histological differences for the definition of separate disease categories.

A further issue affecting IPF research is the polarisation of views around two competing pathogenetic hypotheses. One argues for the primacy of inflammation as the trigger that initiates fibrosis, and the other proposes that fibrosis arises as a consequence of chronic epithelial injury and failure of repair due to aberrant epithelial-mesenchymal interactions. The present authors believe that this schism is hampering understanding of IPF and skewing research priorities.

It is argued here, instead, that abnormalities in multiple pathways involved in wound healing and inflammation lead to the development of idiopathic pulmonary fibrosis, and it is suggested that a new rationale for clinical classification and pathogenesis may be more productive in driving the search for novel therapies in the future.

KEYWORDS: Classification, diagnosis, idiopathic pulmonary fibrosis, nonspecific interstitial pneumonia, pathogenesis

I diopathic pulmonary fibrosis (IPF) is a devastating, progressive fibrotic lung condition that carries a median survival of 2.84.2 yrs, and for which no effective treatments exist [1, 2]. Ongoing scientific and clinical research is crucial if the pathogenesis of IPF is to be understood and effective therapies discovered. However, two controversies are currently hampering efforts to better understand IPF. First, the 2002 American Thoracic Society (ATS)/ European Respiratory Society (ERS) consensus document on the classification of the idiopathic interstitial pneumonias (table 1) has, the present authors believe incorrectly, altered the diagnostic criteria necessary for the diagnosis of IPF [1]. Secondly, two competing pathogenic hypotheses are skewing research priorities. In the present article, both of these issues are addressed and possible solutions suggested.

\section{WHAT IS IPF?}

The ATS/ERS classification defines IPF as "a specific form of chronic fibrosing interstitial pneumonia of unknown aetiology, limited to the lung and associated with the histological entity of usual interstitial pneumonia" [1]. Thus usual interstitial pneumonia (UIP) and IPF are now seen by many as synonymous terms. The most contentious aspect of the new classification was the adoption of the histological lesion of nonspecific idiopathic interstitial pneumonia (NSIP) as a distinct disease. Unlike the other idiopathic interstitial pneumonias, however, NSIP does not exhibit a clinical phenotype that distinguishes it from UIP/IPF. The present authors contend that highlighting subtle histological differences between UIP and NSIP and using these to define separate clinical entities is incorrect and is hindering attempts to better understand the pathogenesis of fibrotic lung disease.

There is strong evidence that different diseases with defined aetiologies may result in the same histological picture. Connective tissue diseases, hypersensitivity pneumonitis, asbestosis and sarcoidosis may progress to cause the histological lesion of UIP [1]. Connective tissue disease, hypersensitivity pneumonitis, drug-induced lung disease, resolved acute respiratory distress
AFFILIATIONS

${ }^{*}$ Centre for Respiratory Research, Rayne Institute, University College London, and

\#Interstitial Lung Disease Unit, Royal Brompton Hospital, London, UK.

CORRESPONDENCE

T.M. Maher

Centre for Respiratory Research

Rayne Institute

University College London

5 University Street

London

WC1E 6JJ

UK

Fax: 442076796973

E-mail: t.maher@ucl.ac.uk

Received:

June 112007

Accepted:

July 162007

STATEMENT OF INTEREST:

None declared. 
syndrome and some cases of cryptogenic organising pneumonia may all produce a histological picture of NSIP $[1,3]$. Fibroblastic foci, the cardinal feature of UIP, also occur, albeit less frequently, in NSIP. Furthermore, UIP and NSIP are frequently found affecting the same individual when lung biopsy samples are taken from different sites in patients with IPF [4].

Overall, the prognosis for patients with NSIP on biopsy is better than that for patients with UIP (median survival 33 versus 56 months) [5]. However, histology is not the sole determinant of disease outcome. LATSI et al. [5] have shown that mortality during the first 2 yrs following diagnosis is primarily linked to severity of pulmonary function impairment and not to histological diagnosis. Furthermore, patients with UIP on biopsy but an indeterminate or NSIP appearance on high-resolution computed tomography (HRCT) have a better prognosis than those with UIP and a typical IPF appearance on HRCT [6]. Another recent observation is that disease progression in IPF, rather than always being gradual and progressive, frequently occurs through a series of rapid stepwise deteriorations [7]. These apparent acute exacerbations of IPF are characterised on biopsy by the appearance of diffuse alveolar damage, the histological lesion of acute interstitial pneumonia [7]. This coexistence of histological patterns and disease behaviour challenges classical thinking concerning the separate and unrelated nature of these conditions (fig. 1).

Familial IPF may manifest as either UIP or NSIP in different members of the same affected family. A recent study of gene expression profiling in UIP and NSIP of either familial or nonfamilial origin found only minor gene expression changes between sporadic UIP and sporadic NSIP cases but much greater variation between familial and nonfamilial cases [8]. In an earlier study, SeLman et al. [9] demonstrated significant gene expression profile differences between UIP and hypersensitivity pneumonitis, but found cases of NSIP with a profile indistinguishable from that of UIP. These data provide compelling evidence for the idea that a single disease entity may be responsible for causing a spectrum of histological abnormality.

The overlap of histological entities, the effects of HRCT appearance and lung function on disease outcome, gene expression profiles and the lack of an NSIP clinical phenotype are strong arguments against limiting the diagnosis of IPF to the histological picture of UIP. The present authors believe that idiopathic UIP and idiopathic NSIP, sharing a common clinical phenotype, form a spectrum of disease with a common pathogenesis. It is their belief that the classification of the idiopathic interstitial pneumonias should, in future, aim to categorise diseases on the basis of common pathogenetic mechanisms. Gene expression profiling offers the tantalising prospect of providing a new gold standard for the diagnosis of IPF. However, given the current absence of a definitive gold standard test, classification should incorporate clinical, radiological and pathological aspects of disease manifestation and behaviour. By relying on this combined approach to classification, it is to be hoped that all points along the phenotypic continuum of disease representing IPF can be recognised, something that is crucial to the ongoing success of both clinical and basic scientific research into this devastating disease.

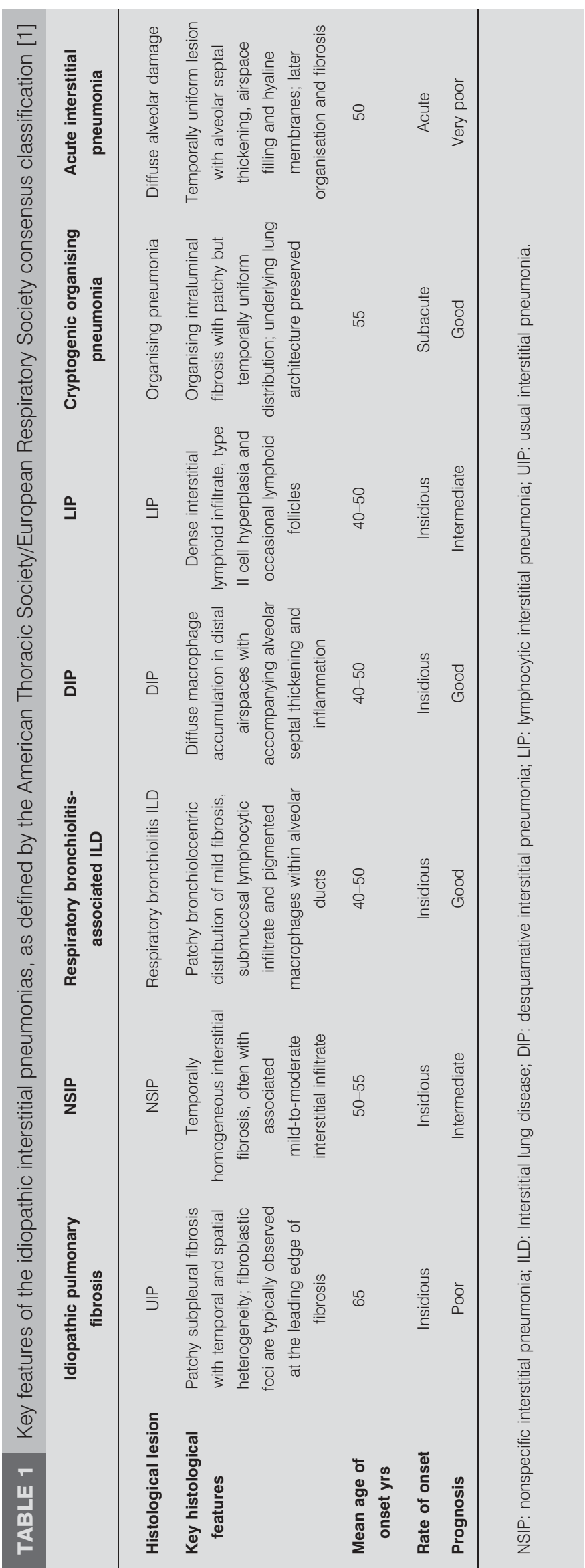




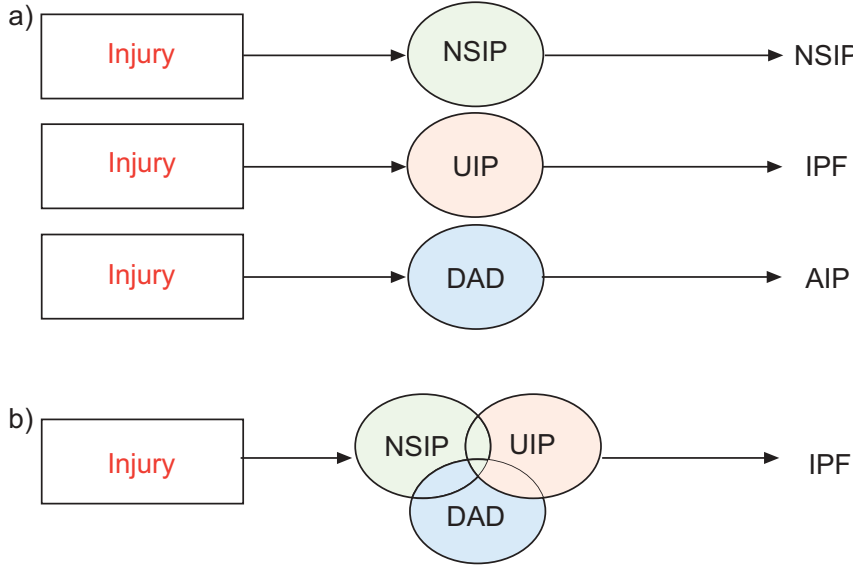

FIGURE 1. a) The paradigm suggested by the current classification system is one of separate injuries giving rise to separate histological lesions and hence separate diseases. b) The present authors propose that the same injury may result in one or a combination of histological lesions that, when sharing a common clinical phenotype, represent the single disease idiopathic pulmonary fibrosis (IPF). NSIP: nonspecific interstitial pneumonia; UIP: usual interstitial pneumonia; DAD: diffuse alveolar damage; AIP: acute interstitial pneumonia.

\section{PROGRESS IN THE UNDERSTANDING OF THE PATHOGENESIS OF IPF}

Since the mid-1980s, remarkable strides have been made in the understanding of the pathogenesis of IPF. Initial pathogenetic hypotheses were shaped largely by concepts developed from studies of wound models. It was believed that, in fibrosis, an exaggerated and uncontrolled healing response occurs, in which the key initiating features are inflammatory cell influx and release of pro-fibrotic products [10]. It was this view that led to the belief that fibrosis could be prevented through inhibition of the inflammatory response, and this rationale still underpins the continued use of corticosteroids and azathioprine as the first-line therapy for IPF [1]. Recently, the argument that inflammatory cells drive fibrosis has come under scrutiny. A growing body of researchers now argue that fibrosis proceeds independently of inflammatory events. Instead, they suggest that fibrosis develops as the consequence of aberrant epithelial and epithelial-mesenchymal responses to chronic alveolar epithelial injury [11].

\section{IPF: MULTIPLE CAUSES AND MULTIPLE PATHWAYS?}

Mammalian defence mechanisms have evolved multiple molecular pathways and mechanisms for the elaboration and resorption of wounds. The process of evolution has created considerable pleiotropism, as well as much redundancy, in cell signalling and wound healing pathways. It is the present authors' belief that IPF develops as a consequence of abnormalities occurring in multiple biological pathways that affect inflammation and wound repair. A consequence of evolutionary adaptation is that major abnormalities in single key molecules can be compensated for by the recruitment of otherwise redundant mechanisms. It is often only when errors occur in multiple pathways that protective mechanisms are overcome and disease develops. The idea of multiple hits has best been elucidated in malignancy, where it has been demonstrated that somatic mutations in a sequence of key oncogenes are required before malignancy develops [12]. Even in the best-characterised malignancies, the sequence of mutations that result in the same disease varies between individuals [13]. Furthermore, in a number of different cancers, this variation has been shown to affect the response to specific anticancer drugs [14].

It is now clear that both individual susceptibility to IPF and the pattern of disease behaviour and progression are linked to patient genotype [15]. A number of investigators have undertaken an extensive search for fibrosis genes. A range of polymorphisms have been related to susceptibility to IPF, and to disease severity and progression [15]. Furthermore, tentative evidence has been published suggesting that somatic gene mutation plays a role in the development of IPF [16, 17].

The trigger that initiates the development of fibrosis in IPF remains unknown. However, there is increasing evidence that a wide range of potentially injurious factors may play a role in the initiation and progression of IPF $[18,19]$. A disproportionate number of patients with IPF have smoked heavily [1]. Oxidative stress, environmental pollutants and dusts have all been implicated as potential causes of IPF [18]. Studies of viral respiratory tract infection in patients with IPF suggest an increased prevalence of past infection [19]. Furthermore, there is some suggestion that IPF patients have suffered infection with specific subtypes of virus particularly associated with the induction of somatic mutation [19].

Following injury, the present authors propose that a multiplepathway mechanism is at play in the pathogenesis of IPF (fig. 2). A range of molecular abnormalities that can result in fibrosis are now well characterised. The clotting cascade, antioxidant pathways, apoptosis, inflammatory cytokines, angiogenesis and vascular remodelling, growth factors, surfactant and matrix regulatory factors have all been implicated

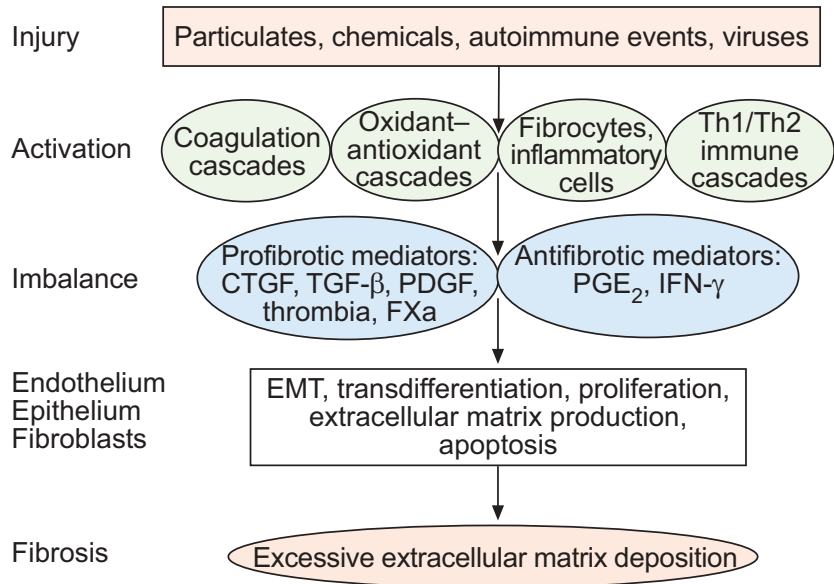

FIGURE 2. A new model for the pathogenesis of idiopathic pulmonary fibrosis: injury activates multiple inflammatory, cell signalling and repair pathways. Activation of these cascades causes an imbalance in pro- and antifibrotic mediators. In turn, these mediators activate multiple cell types, causing changes in cellular functioning and cell-cell interactions that ultimately result in progressive fibrosis. Th: T-helper cell; CTGF: connective tissue growth factor; TGF- $\beta$ : transforming growth factor- $\beta$ PDGF: platelet-derived growth factor; FXa: factor Xa; PG: prostaglandin; IFN- $\gamma$ : interferon- $\gamma$; EMT: epithelial-mesenchymal transition. 
in fibrosis in animal models [20-23]. Furthermore, these cascades initiate changes in the behaviour and morphology of multiple cell types, including epithelial cells, fibroblasts, endothelial cells, resident and migratory inflammatory cells, and, as has recently been described, circulating fibroblast progenitor cells (fibrocytes) [20-25]. Evidence for the relative roles of these key pathways and the interactions between different cell types in the pathogenesis of fibrosis in humans is gradually emerging [20-25].

As is the case in other complex diseases, the present authors believe that the balance of abnormalities in each of these key pathways may vary between affected individuals. This would explain the range of clinical, radiological and pathological phenotypes observed in IPF. Current thinking in IPF has tended to focus on individual mediators of fibrosis or single pathways linked to fibrogenesis. The limitations of this model have been reflected in disappointing trials of therapy targeting individual disease pathways [26, 27]. Ongoing adherence to this approach threatens to affect the development of future therapies and therapeutic strategies. The present authors believe that adopting a multiple-pathway model for understanding the pathogenesis of IPF would facilitate a future research approach better suited to the understanding of this complex disease. Furthermore, if the multiple-pathway hypothesis is correct, it seems likely that the most effective approach to treatment would be to target multiple fibrosis pathways simultaneously. This approach to treatment is analogous to that used in the treatment of many cancers. Furthermore, as with many cancers, individualisation of treatment on the basis of each patient's predominant pathogenetic pathway may become possible as a deeper understanding of the balance of abnormalities that result in pulmonary fibrosis is developed.

\section{CONCLUSION}

Idiopathic pulmonary fibrosis is a devastating condition. Clear classification of idiopathic pulmonary fibrosis is hindered by the lack of a gold standard test. Future attempts to classify the idiopathic interstitial pneumonias should take into account clinical and radiological manifestations of disease rather than relying simply on subtle histological differences as a basis for the definition of separate disease entities. An understanding of the full spectrum of disease encompassed by idiopathic pulmonary fibrosis would facilitate better understanding of its pathogenesis. However, the dogmatic insistence that a single pathway holds the key to the pathogenesis of idiopathic pulmonary fibrosis represents a barrier to the future success of research into idiopathic pulmonary fibrosis, and is likely to hinder the ongoing search for treatments. A multiple-pathway model of disease pathogenesis better explains the currently available evidence and provides a rationale for adopting an approach to treatment that targets multiple fibrosis cascades simultaneously.

\section{REFERENCES}

1 American Thoracic Society, European Respiratory Society. American Thoracic Society/European Respiratory Society international multidisciplinary consensus classification of the idiopathic interstitial pneumonias. Am J Respir Crit Care Med 2002; 165: 277-304.

2 Bjoraker JA, Ryu JH, Edwin MK, et al. Prognostic significance of histopathological subsets in idiopathic pulmonary fibrosis. Am J Respir Crir Care Med 1998; 157: 199-203.

3 Nicholson AG, Wells AU. Nonspecific interstitial pneumonia - nobody said it's perfect. Am J Respir Crit Care Med 2001; 164: 1553-1554.

4 Monaghan H, Wells AU, Colby TV, du Bois RM, Hansell DM, Nicholson AG. Prognostic implications of histologic patterns in multiple surgical lung biopsies from patients with idiopathic interstitial pneumonias. Chest 2004; 125: 522-526.

5 Latsi PI, du Bois RM, Nicholson AG, et al. Fibrotic idiopathic interstitial pneumonia: the prognostic value of longitudinal functional trends. Am J Respir Crit Care Med 2003; 168: 531-537.

6 Flaherty KR, Thwaite EL, Kazerooni EA, et al. Radiological versus histological diagnosis in UIP and NSIP: survival implications. Thorax 2003; 58: 143-148.

7 Kim DS, Park JH, Park BK, Lee JS, Nicholson AG, Colby T. Acute exacerbation of idiopathic pulmonary fibrosis: frequency and clinical features. Eur Respir J 2006; 27: 143-150.

8 Yang IV, Burch LH, Steele MP, et al. Gene expression profiling of familial and sporadic interstitial pneumonia. Am J Respir Crit Care Med 2007; 175: 45-54.

9 Selman M, Pardo A, Barrera L, et al. Gene expression profiles distinguish idiopathic pulmonary fibrosis from hypersensitivity pneumonitis. Am J Respir Crit Care Med 2006; 173: 188-198.

10 Gauldie J, Jordana M, Cox G. Cytokines and pulmonary fibrosis. Thorax 1993; 48: 931-935.

11 Selman M, King TE, Pardo A. Idiopathic pulmonary fibrosis: prevailing and evolving hypotheses about its pathogenesis and implications for therapy. Ann Intern Med 2001; 134: 136-151.

12 Benito M, Diaz-Rubio E. Molecular biology in colorectal cancer. Clin Transl Oncol 2006; 8: 391-398.

13 Colebatch A, Hitchins M, Williams R, Meagher A, Hawkins NJ, Ward RL. The role of MYH and microsatellite instability in the development of sporadic colorectal cancer. Br J Cancer 2006; 95: 1239-1243.

14 Pao W, Wang TY, Riely GJ, et al. KRAS mutations and primary resistance of lung adenocarcinomas to gefitinib or erlotinib. PLoS Med 2005; 2: e17.

15 Lawson WE, Lloyd JE. The genetic approach in pulmonary fibrosis: can it provide clues to this complex disease? Proc Am Thorac Soc 2006; 3: 345-349.

16 Vassilakis DA, Sourvinos G, Spandidos DA, Siafakas NM, Bouros D. Frequent genetic alterations at the microsatellite level in cytologic sputum samples of patients with idiopathic pulmonary fibrosis. Am J Respir Crit Care Med 2000; 162: 1115-1119.

17 Mori M, Kida H, Morishita $\mathrm{H}$, et al. Microsatellite instability in transforming growth factor- $\beta 1$ type II receptor gene in alveolar lining cells of idiopathic pulmonary fibrosis. Am J Respir Cell Mol Biol 2001; 24: 398-404.

18 Selman M, Pardo A. Role of epithelial cells in idiopathic pulmonary fibrosis: from innocent targets to serial killers. Proc Am Thorac Soc 2006; 3: 364-372. 
19 Doram P, Egan JJ. Herpesviruses: a cofactor in the pathogenesis of idiopathic pulmonary fibrosis? Am J Physiol Lung Cell Mol Physiol 2005; 289: L709-L710.

20 Chambers RC. Role of coagulation cascade proteases in lung repair and fibrosis. Eur Respir J 2003; 22: Suppl. 44, 33s-35s.

21 Kinnula VL, Fattman CL, Tan RJ, Oury TD. Oxidative stress in pulmonary fibrosis: a possible role for redox modulatory therapy. Am J Respir Crit Care Med 2005; 172: 417-422.

22 Thannical VJ, Horowitz JC. Evolving concepts of apoptosis in idiopathic pulmonary fibrosis. Proc Am Thorac Soc 2006; 3: 357-363.

23 Keane MP, Strieter RM, Belperio JA. Mechanisms and mediators of pulmonary fibrosis. Crit Rev Immunol 2005; 25: 429-463.
24 Willis BC, du Bois RM, Borok Z. Epithelial origin of myofibroblasts during fibrosis in the lung. Proc Am Thorac Soc 2006; 3: 377-382.

25 Mehrad B, Burdick MD, Zisman DA, Keane MP, Belperio JA, Strieter RM. Circulating peripheral blood fibrocytes in human fibrotic interstitial lung disease. Biochem Biophys Res Commun 2007; 353: 104-108.

26 Raghu G, Brown KK, Bradford WZ, et al. A placebo controlled trial of interferon gamma- $1 \mathrm{~b}$ in patients with idiopathic pulmonary fibrosis. N Engl J Med 2004; 350: 125-133.

27 King TE, Behr J, Brown KK, du Bois RM, Raghu G. Bosentan use in idiopathic pulmonary fibrosis (IPF): results of the placebo controlled BUILD-1 study. Am J Respir Crit Care Med 2006; 3: A524. 